\title{
Las lesiones cerebrales asintomáticas se asocian a un aumento de la incidencia de accidente cerebrovascular y de la mortalidad global
}

Asymptomatic brain injuries are associated with an increased incidence of stroke and overall mortality

\section{Objetivos}

Evaluar el riesgo de accidente cerebrovascular (ACV) y la mortalidad global en individuos con lesiones cerebrales subclínicas y con sectores de la sustancia blanca con señal hiperintensa detectados por resonancia.

\section{Diseño, lugar y pacientes}

Estudio de cohorte prospectiva de base comunitaria (Atherosclerosis Risk in Communities Study, en inglés ARIC) llevado a cabo en Estados Unidos: condados de Forsyth (North Carolina) y Jackson (Mississippi). Fueron seguidos un promedio de 14,5 años 1884 adultos de 50 a 73 años sin antecedentes clínicos de ACV.

\section{Medición de resultados principales}

Las lesiones lacunares fueron clasificadas de acuerdo a su tamaño
Windham BG y col. Annals of Internal Medicine. 2015;163:22-31 (mayores y menores a $3 \mathrm{~mm}$ ); y los aumentos de la intensidad de la sustancia blanca, mediante una escala de 0 a 9 puntos. Fueron estimadas la incidencia de ACV, la mortalidad por todas las causas y la mortalidad específica por ACV, ajustadas por edad, sexo, raza, nivel educativo, índice de masa corporal, tabaquismo, consumo de alcohol, diabetes, presión arterial sistólica y diastólica, uso de medicación antihipertensiva, enfermedades cardiovasculares, niveles de colesterol y triglicéridos, y uso de estatinas. Para el análisis multivariable se usó un modelo de riesgos proporcionales de Cox.

\section{Resultados principales}

La tabla 1 describe la asociación entre el tipo de lesiones y el riesgo ajustado de ACV y muerte de acuerdo a los principales hallazgos en la resonancia nuclear.

Tabla 1. asociación entre el tipo de lesión y el riesgo de ACV y muerte.

\begin{tabular}{|c|c|c|c|}
\hline & & $\begin{array}{c}\text { ACV } \\
\text { HR (IC 95\%) }\end{array}$ & $\begin{array}{l}\text { Mortalidad } \\
\text { HR (IC 95\%) }\end{array}$ \\
\hline \multirow[t]{3}{*}{ Lesiones en el sistema nervioso central } & Ausencia de lesiones & \multicolumn{2}{|c|}{ Referencia } \\
\hline & Menores a 3 mm & $3,47(1,86$ a 6,49$)$ & $3,05(1,04 a \mathrm{a} 8,94)$ \\
\hline & Cualquier tipo de lesión & $8,59(4,69$ a 15,73$)$ & $6,97(2,03$ a 23,93$)$ \\
\hline \multirow[t]{2}{*}{ Señal hiperintensa en la sustancia } & Puntaje menor a 3 & \multicolumn{2}{|c|}{ Referencia } \\
\hline & Puntaje mayor a 3 & $2,14(1,45$ a 3,16) & $1,78(1,42$ a 2,23$)$ \\
\hline
\end{tabular}

ACV: accidente cerebrovascular; HR: hazard ratio; IC 95\%: intervalo de confianza del 95\%.

\section{Conclusiones}

Las lesiones pequeñas en el sistema nervioso central y la hiperintensidad de la sustancia blanca se asocian con un aumento del riesgo de ACV y muerte.

Fuente de financiamiento: National Heart, Lung and Blood Institute.

\section{Comentario}

Existe suficiente evidencia ${ }^{1,2,3}$ para afirmar que, en personas asintomáticas, la aparición de lesiones en la sustancia blanca ${ }^{4}$ o infartos ${ }^{5}$ se comportan como un factor de riesgo independiente para padecer un ACV y, que incluso aumentarían la probabilidad de desarrollo de demencia. Vale destacar que en este estudio se observó que la mayoría de los pacientes que tenían lesiones en el sistema nervioso central eran de raza negra y sufrían hipertensión arterial. Entre sus limitaciones, mencionamos que fueron evaluados pocos casos de ACV (espe- cialmente hemorrágicos) y pocos participantes con lesiones menores a $3 \mathrm{~mm}$ o bilaterales.

\section{Conclusiones de la comentadora}

Si bien en la práctica clínica diaria se tiende a minimizar este tipo de lesiones, consideramos importante pesquisar en estos pacientes los factores de riesgo cardiovascular modificables para optimizar su control y minimizar la probabilidad de desarrollo de eventos cardio y cerebro-vasculares.

Marina Romano [ Jefa Neurología Vascular. Centro de Educación Médica e Investigaciones clínicas Norberto Quirno (CEMIC). romano.marina@gmail.com ]

Romano M. Las lesiones cerebrales asintomáticas se asocian a un aumento de la incidencia de accidente cerebrovascular y de la mortalidad global. Evid Act Pract Ambul 2016;19(4):116. Comentado de: Windham B y col. Small Brain Lesions and Incident Stroke and Mortality: A Cohort Study. Ann Intern Med. 2015;163(1):22-31. PMID: 26148278.

\section{Referencias}

1. Fisher C. Lacunar strokes and infarcts: a review. Neurology. 1982;32:871-6.

2. Bernick C y col. Cardiovascular Health Study Collaborative Re- search Group. Silent MRI infarcts and the risk of future stroke: the Cardiovascular Health Study. Neurology. 2001;57:1222-9.

3. Bokura $\mathrm{H}$ y col. Silent brain infarction and subcortical white matter lesions in- crease the risk of stroke and mortality: a prospective cohort study. J Stroke Cerebrovasc Dis. 2006;15:57-63.

4. Buyck J y col. Cerebral white matter lesions are associated with the risk of stroke but not with other vascular events: the 3-City Dijon Study. Stroke. 2009;40:2327-31.

5. Longstreth W. Lacunar infarcts defined by magnetic resonance imaging of 3660 elderly people: the Cardiovascular Health Study. Arch Neurol. 1998; 55:1217-25. 